\title{
Franz Kafka und Albert Bassermann
}

Unter den vielen schwer verständlichen Mitteilungen Kafkas ist wohl die an Max Brod auf einer Kunstpostkarte ${ }^{1}$ vom 9. Dezember 1910 eine der rätselhaftesten. Sie lautet:

Max, ich hab eine Hamletaufführung gesehn oder besser[:] den Bassermann gehört. Ganze Viertelstunden hatte ich bei Gott das Gesicht eines andern Menschen, von Zeit zu Zeit mußte ich von der Bühne weg in eine leere Loge schauen, um in Ordnung zu kommen.

Dein Franz. ${ }^{2}$

Drei Tage zuvor hatte Kafka auf einer Reise nach Weimar einen Abstecher nach Berlin gemacht und dort eine Aufführung von Shakespeares Hamlet besucht mit dem Schauspieler Albert Bassermann in der Titelrolle. Das Stück mit seinem von den väterlichen Ansprüchen überforderten Protagonisten dürfte ihm kaum gleichgültig gewesen sein. Dennoch verliert er kein Wort über dieses Vater-Sohn-Drama der Weltliteratur par excellence. Das mag darin begründet sein, dass er, wie sein Freund Brod wohl auch, mit dem Drama längst vertraut war. ${ }^{3}$ Auch über die in der Theatergeschichte Epoche machende Inszenierung Max Reinhardts schweigt er sich aus. Die Postkarten-Mitteilung an Brod sollte vermutlich auch keine Theaterkritik darstellen und erst recht nicht einer Gesamtbeurteilung des Theaterbesuchs vorgreifen. Wenn es Kafka darum gegangen wäre, eine solche zu liefern, so hätte er sie ja später dem Freund mündlich in Prag nachliefern können. Freilich ist bei solchen Spekulationen ${ }^{4}$ Vorsicht geboten, denn Gesamturteile, Einschätzungen des Ganzen, besitzen bei Kafka Seltenheitswert oder

1 Mit einer Wiedergabe des von Goethe gezeichneten Schlosses Kochberg, dem ehemaligen Landsitz der Familie von Stein.

2 Brief vom 9. Dezember 1910, in: Franz Kafka: Briefe 1900-1912, hg. von Hans-Gerd Koch, Frankfurt a.M. 1999, S. 129.

3 Was Kafka betrifft, vgl. Hartmut Binder: Kafka-Handbuch in zwei Bänden, Bd. 1, Stuttgart 1979, S. 293, sowie Peter-André Alt: Franz Kafka. Der ewige Sohn, München 2005, S. 199 und 439f.; was Brod angeht, vgl. Max Brod: Streitbares Leben 1884-1968, München u.a. 1969, S. 129.

4 Reiner Stach bringt eine andere, meines Erachtens abwegige vor: „Hätte Brod ihn gefragt, ob denn neben dem Theaterstar Albert Bassermann, der gestisch wie stimmlich exzessiv 
genauer: Er geht ihnen aus dem Wege und demonstriert sein vorgebliches Desinteresse, indem er sich an ausgefallene Details, an geradezu absonderlich scheinende Nebensächlichkeiten hält und diese derart ins Zentrum rückt, dass sie sich jeglicher Hierarchisierungsmöglichkeit im Hinblick auf ein Ganzes entziehen. ${ }^{5}$

Statt von Stück und Inszenierung ist also nur vom Hauptdarsteller die Rede. Diese doppelte Reduktion der Hamlet-Aufführung mag dem Ruhm des damals schon sehr bekannten Schauspielers geschuldet sein. ${ }^{6}$ Der 1867 in Mannheim geborene Albert Bassermann hatte sich als Charakterdarsteller des Naturalismus unter Otto Brahm einen Namen gemacht, bevor er bei Reinhardt am Deutschen Theater reüssierte. In Siegfried Jacobsohns Schaubühne hat Harry Kahn (1883-1970) die Hamlet-Aufführung rezensiert und damit ein schriftliches Dokument hinterlassen, das uns - zusammen mit einer Fotografie des Schauspielers in der Titelrolle (Abb. 1) ${ }^{7}$ - als Ersatz für das optisch wie akustisch verloren gegangene Ereignis dienen kann.

Kahn führt uns Bassermann zuerst als ruhende Gestalt vor Augen: »dunkel, den scharfzügigen, blond-und-bleichen Kopf in trotzigem Gram auf den hohen Rumpf gesenkt, in einer drohenden Geschlossenheit und Abgeschlossenheit«. ${ }^{8}$ Dann beschreibt er den Schauspieler in Erregung und verbindet seine pathognomischen Befunde mit einer Deutung, die schließlich in eine Bewertung übergeht:

Und Bassermann rast, überschlägt und überschreit sich; seine Füße tragen ihn nicht mehr, seine Stimmbänder zerreißen schier, sein Mund, seine Augen werden maskenstarr, wenn er zum ersten Mal das ganze Gewebe durchschaut und erkennt, was ihm das Schicksal angetan hat. [...] Worte und Gebärden unförmig und unge-

agierte, auch die Inszenierung gut war, wäre Kafka wohl in Verlegenheit geraten." Vgl. Reiner Stach: Kafka. Die frühen Jahre, Frankfurt a.M. 2014, S. 368.

5 Für Kafkas physiognomische Beschreibungen vgl. Peter von Matt: ... fertig ist das Angesicht. Zur Literaturgeschichte des menschlichen Gesichts, Frankfurt a.M. 1989, S. 24-50. Die hier skizzierte Beschreibungstechnik findet sich jedoch, wie ich demnächst zeigen möchte, auch sonst bei Kafka und betrifft ganz allgemein seine Einschätzung der Beziehung von Teil und Ganzem.

6 Meine These wäre, dass die doppelte Reduktion die Rezipienten geradezu nötigen soll, die Vorstellung von einem Ganzen, nämlich von dem, wie das Stück Shakespeares und die Inszenierung Reinhardts nun wirklich insgesamt gewesen seien, im eigenen Innern zu suchen. Siehe hierzu die folgenden Überlegungen.

7 Kafka hatte die Hamlet-Aufführung mit Bassermann am 6. Dezember 1910 im Deutschen Theater besucht. Für die Genehmigung zur Veröffentlichung danke ich Stephan Dörschel, Abteilungsleiter des Archivs Darstellende Kunst, Akademie der Künste, Berlin.

8 Harry Kahn: Der neue Hamlet, in: Die Schaubühne 6 (1910), Nr. 48, 1. Dezember 1910, S. 1235-1236, hier S. 1235. 
formt, glühend und roh gleich Lava aus dem Krater, zu speien, auszuwürgen, dieser Naturalismus nicht so sehr des Gefühls wie des Intellekts - hier hat er einmal innerlichste Berechtigung und Bedeutung; hier ist er nicht Analyse, sondern dient der Synthese einer Gestalt. Was will es da besagen, daß Bassermann manchmal Sätze bis zur Sinnlosigkeit zertrennt, zerpflückt, andre ebenso zusammenkuppelt oder ineinanderknüllt; was will es besagen, daß er meist mehr Mannheimer als Helsingörer [...] ist. [...] Alle Schwächen und Uebertreibungen fallen ab vor der Einheit und Reinheit der ganzen Gestalt. ${ }^{9}$

Der expressive, ja expressionistisch hoch gestimmte Ton kann nicht verbergen, dass der Rezensent einem relativ schlichten Beschreibungsverfahren folgt. Kahn glaubt durch Anhäufung möglichst vieler Einzelheiten die "ganze Art Bassermanns «10 erfassen zu können. Für sie verwendet er zweimal den Begriff »Gestalt«, der für die "Synthese« und die »Einheit« aller physiognomisch und pathognomisch ermittelten Daten einstehen soll ${ }^{11}$ und der auch für Kafka bedeutungsvoll wird, wenn dieser ihn auch, wie sich zeigen wird, anders fasst als der Schaubühnen-Rezensent.

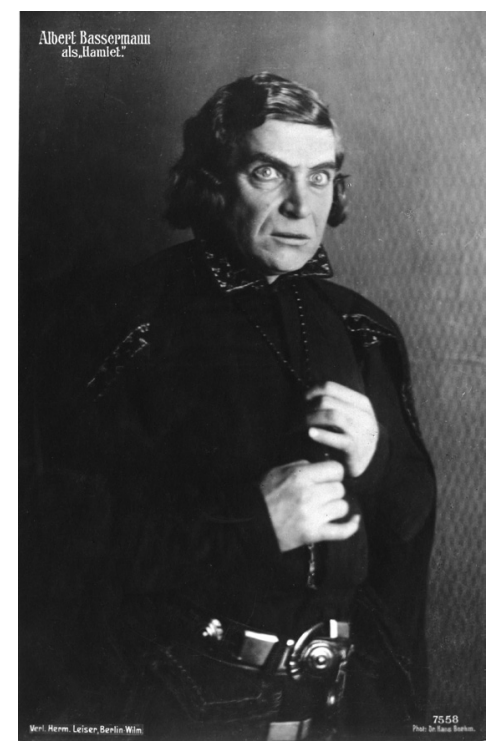

Abb. 1: Albert Bassermann als Hamlet unter der Regie von Max Reinhardt, Fotografie: Dr. Hans Boehm, (C) Akademie der Künste, Berlin (Sign. FSTH 514)

9 Ebd., S. $1235 f$.

10 Ebd., S. 1236.

11 Siehe das Zitat bei ebd., S. 1235. 
Überhaupt verfährt Kafka völlig anders als Kahn. Er teilt mit ihm einzig die Hochschätzung der Schauspielkunst Bassermanns, die alle anderen künstlerischen Leistungen, selbst die des Regisseurs Reinhardt, überstrahle. Kafka beschreibt gar nichts - weder das Aussehen, die Gestik und Mimik, noch die Stimme. Er spricht nur von der Wirkung, die Bassermann auf ihn ausgeübt hat. Dabei bleibt freilich unklar, warum er sich korrigiert. Warum findet er es »besser« zu schreiben, er habe den Bassermann gehört, als zu sagen, er habe ihn oder die Inszenierung mit ihm gesehen? Wenn, wie Kafka suggeriert, die Darstellung Bassermanns vor allem ein stimmliches Ereignis gewesen sein soll, warum hat sich der Theaterbesucher von der Bühne abgewendet und "in eine leere Loge" geblickt? Mit einer solchen Geste wird er wohl kaum in die Lage versetzt worden sein, Bassermann zu überhören. Wenn der Theaterbesucher sich durch sein Wegschauen immer wieder "in Ordnung" bringen wollte oder gar konnte, dann müsste die Unordnung, in die er phasenweise geraten war, wohl eine Folge des Sichtbaren gewesen, also eher durch Mimik und Gestik hervorgerufen worden sein.

Dass sich das eigene »Gesicht« durch das Gesehene verändert haben könnte, dafür spricht der erinnernde Rückblick auf das Berliner Theatererlebnis mehr als zwei Jahre später. Im März 1913 sieht Kafka nämlich Bassermann erneut, und zwar auf einem Plakat des Stummfilms Der Andere; und er wird, wie er an Felice Bauer schreibt, "wieder ergriffen, wie damals in Berlin «. ${ }^{12}$ Er zerrt seine Freunde »immer wieder" vor das Plakat und die filmstills ${ }^{13}$ (Abb. 2 und 3). ${ }^{14}$

Brief an Felice Bauer vom 4./5. März 1913, in: Franz Kafka: Briefe 1913-März 1914, hg. von Hans-Gerd Koch, Frankfurt a.M. 2001, S. 120-122, hier S. 121. Vgl. auch Hanns Zischler: Kafka geht ins Kino, Reinbek bei Hamburg 1998, S. 96, der zum ersten Mal die relevanten Materialien über Kafkas Beziehungen zum Film zusammengetragen hat. Über Bassermann und den Film Der Andere vgl. die Zusammenstellung ebd., S. 96-104.

Dabei handelt es sich um Publicity Stills. Bei solchen "fotografiert ein auf dem Filmset anwesender Fotograf Situationen während des Drehs und lässt zuweilen auch die Darsteller eigens für seine Kamera posieren. Diese abseits der eigentlichen Dreharbeiten entstehenden Fotos zirkulieren denn auch bereits, selbst wenn der Film, den sie bewerben, noch gar nicht fertig gedreht ist, und es sind diese Publicity Stills, die man auch später als Aushangbilder in den Foyers der Kinos und auf Plakaten sehen wird «. Johannes Binotto: Optisch Unbewusstes. Zu den entwundenen Film-Bildern von Mattias Gabi [=Essay zur Ausstellung Frame von Matthias Gabi im Raum für zeitgenössische Fotografie, Volkart Stiftung Winterthur, 30. Januar bis 21. März 2015], www.coalmine.ch/wordpress/wp-content/ upload/2014 (28. September 2015).

14 In dem Film, der nach dem gleichnamigen Stück von Paul Lindau gedreht wurde und der sich an Robert Louis Stevensons The strange case of Dr. Fekyll and Mr. Hyde von 1886 anlehnt, spielt Bassermann den Protagonisten, der an einer Bewusstseinsspaltung leidet. Am Tag ein angesehener Rechtsanwalt, verwandelt er sich des Nachts in einen Verbrecher. Kafka sah den Film wie die Filmstills im Vorraum des Prager Kinematografentheaters 


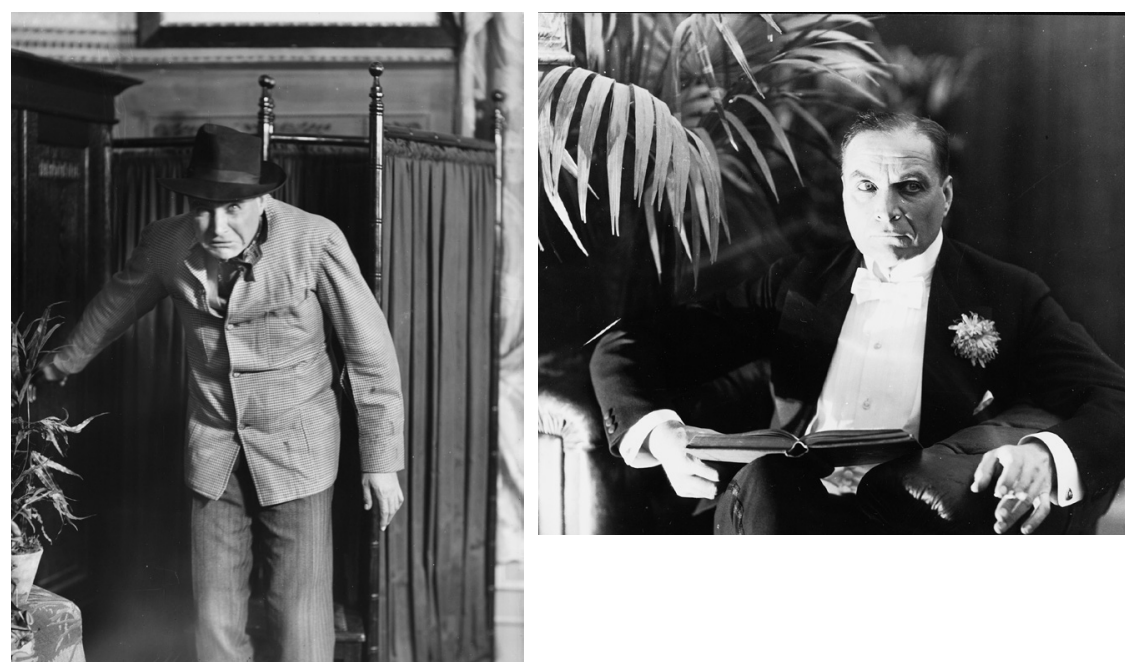

Abb. 2 und 3: Albert Bassermann im Film Der Andere (Regie: Max Mack, Deutschland 1913) als Einbrecher (Abb. 2) und als Rechtsanwalt Dr. Hallers (Abb. 3),

(C) Deutsche Kinemathek, Berlin

Obwohl er - anders als Bassermann selbst ${ }^{15}$ - den Film als missraten ansah, ${ }^{16}$ sei er von diesem Schauspieler aufs Neue "ein wenig verwandelt « worden. ${ }^{17}$ Die Erwähnungen Bassermanns in den Briefen von 1913 legen also nahe,

Lucerna Anfang März 1913. »Auf einem Plakat, wo B. allein im Lehnstuhl abgebildet war, hat er mich wieder ergriffen, wie damals in Berlin« (Franz Kafka: Briefe 1913-März 1914, S. 121). Für die Publikationsgenehmigung der Abbildungen 2 und 3 danke ich der Leiterin des Fotoarchivs der Deutschen Kinemathek, Julia Riedel, in Berlin.

15 Bassermann kann keinen wesentlichen Unterschied zwischen dem neuen Medium und einer Theateraufführung feststellen; der Film habe lediglich den Vorteil, die Kunst eines Schauspielers für alle Zeiten aufbewahren zu können. Über sich in der Rolle des Protagonisten Haller wie auch über den Film insgesamt äußert er sich sehr zufrieden: »Vorderhand seien wir froh über das Erreichte." Albert Bassermann: Wie ich mich im Film sehe (Drehbericht), in: Lichtbild-Bühne Nr. 5, 1. Februar 1913. - Der Bericht erschien dann noch einmal mit dem gleichen Titel ohne den Klammerzusatz in: Deutsche Zeitung. Bohemia, Nr. 67, Sonntags-Beilage 9. März 1913, S. 33-34, hier S. 34. - Die Bekanntschaft mit diesem Text verdanke ich Jiskra Jindrová, Prag.

16 »Von Bassermann könnte ich Dir sehr viel erzählen, so elend das Stück ist, und so sehr Bassermann darin mißbraucht wird und sich selbst mißbraucht." Franz Kafka: Briefe 1913-März 1914, S. 134-135, hier S. 135.

17 Ebd. Peter-André Alt betont bei seiner Inhaltsangabe des Films mit Recht, dass Kafka in Anbetracht des Sujets, das ja von unbewussten, krankhaften Verwandlungen eines Rechtsanwalts in einen Verbrecher handelt, eine »durchaus ironische Formulierung " gebrauche. Peter-André Alt: Kafka und der Film. Über kinematographisches Erzählen, München 2009, S. 109. 
dass der ungeheure Eindruck in Berlin vor allem durch den Sehsinn zustande gekommen sei und dass das "Gesicht eines andern Menschen« das des Hamlet-Darstellers Albert Bassermann gewesen sein muss.

Freilich werden mit diesen beiden Hypothesen die Briefkarte an Brod eher noch rätselhafter und die Korrektur Kafkas - »oder besser[:] den Bassermann gehört« - noch weniger verständlich. Der Fortgang der Mitteilung muss verwirren, denn beim Umstieg vom Visuellen auf das Auditive bleibt es nicht. Der Kartenschreiber kehrt sogleich wieder zum Optischen zurück, indem er vom »Gesicht« (was immer auch darunter verstanden sein mag) eines andern Menschen und vom "Schauen« in eine leere Loge spricht. Auch was mit der Bezeichnung »in Ordnung kommen" gemeint ist, bleibt unklar.

Es existieren hier also zwei Problemkreise, die es zu verstehen gilt und die auf spannungsvolle Weise miteinander verbunden sind. Sie sollen hier der Reihe nach behandelt werden, damit ihr Zusammenhang ersichtlich werden kann. Der eine ist das Verhältnis von Subjekt und Objekt, das nicht antithetisch strukturiert, sondern durch Übergänglichkeit und Anverwandlung gekennzeichnet ist. Der andere betrifft das Verhältnis der Sinne zueinander und ihr je spezifischer Beitrag bei der Herstellung eines Gesamtbilds. Für die Erörterung beider Problemkreise reicht es nicht aus, Kafkas Kartengruß aus Berlin mikroanalytisch zu betrachten; und es genügt ebenso wenig, die Differenzen zwischen den verschiedenen Sinneswahrnehmungen allein als ontologische Gegebenheiten zu fassen. Auch wenn es im Folgenden vor allem um die Postkarte an Brod geht und ich immer wieder auf sie zurückkommen möchte, sollen doch auch andere Texte Kafkas miteinbezogen werden. Und auch wenn ich anthropologische Grundtatsachen berühre, so gehe ich doch davon aus, dass sich die menschliche Sinneswahrnehmung innerhalb großer historischer Zeiträume verändern kann. ${ }^{18}$ In einem ersten Teil soll das Problem der Anverwandlung, also das der Übernahme des "Gesichts eines andern", diskutiert werden. Im zweiten Teil gehe ich dann detailliert auf das Verhältnis der beiden Sinnesvermögen Gesicht und Gehör ein und zeige, wie ihre Leistungen im Begriff der `Gestaltqualität‘ konvergieren.

18 Vgl. hierzu Benjamins Bemerkung: »Innerhalb großer geschichtlicher Zeiträume verändert sich mit der gesamten Daseinsweise der menschlichen Kollektiva auch die Art und Weise ihrer Sinneswahrnehmung." Walter Benjamin: Das Kunstwerk im Zeitalter seiner technischen Reproduzierbarkeit, in: ders.: Gesammelte Schriften, hg. von Rolf Tiedemann und Hermann Schweppenhäuser, Bd. I.2, Frankfurt a.M. 1974, S. 431-508, hier S. 478. 
1.

Franz Kafka hat immer wieder eingestanden, dass er Personen, die ihn beeindruckten, ja sogar solche, die ihm zu schaffen machten, nachgeahmt oder ihr "Gesicht" angenommen habe. Zu den Personen, denen er sich anverwandelte, gehören neben Bassermann auch andere Schauspieler, ${ }^{19}$ Alexander Moissi ${ }^{20}$ etwa, oder Berühmtheiten wie Napoleon, ${ }^{21}$ aber auch Personen aus seinem privaten Umkreis wie sein Vater, ${ }^{22}$ sein Schwager Karl Hermann Kafka, sein Freund Max Brod ${ }^{23}$ oder sein Vorgesetzter in der Arbeiter-UnfallVersicherungs-Anstalt Eugen Pfohl. ${ }^{24}$ Alle diese Personen werden nicht oder jedenfalls nicht ausschließlich in ihrem Äußeren nachgeahmt, ${ }^{25}$ vielmehr eignet sich Kafka vor allem ihre Physiognomie insgesamt - oder um den noch zu erörternden Terminus zu benützen: ihre `Gestaltqualität $<$ - an. Am detailliertesten wird der Vorgang der Anverwandlung in einer Tagebuchaufzeichnung vom Herbst 1911 analysiert. Nach einer Begegnung mit Kurt Tucholsky und dessen Buchillustrator Kurt Szafranski notiert sich Kafka:

19 Die Nachahmung von Schauspielern lag für Kafka vielleicht deshalb besonders nahe, weil diese sich ihm ja auch in zweierlei Gestalt offenbarten; nicht unbedingt, wie er selbst, als "etwas" und »nichts" - siehe weiter unten das Zitat aus dem zitierten Brief an Felice Bauer vom 4./5. März 1913 -, sondern als darstellende Person und dargestellte Figur.

Einem Tagebucheintrag vom 3. März 1912 zufolge verstärkt Moissis Redeweise »sich nach der ihm mitgegebenen Anlage und schließt uns ein. - Die Möglichkeit der eigenen Stimme[,] die man hier sieht«. Franz Kafka: Tagebücher, hg. von Hans-Gerd Koch u.a., Frankfurt a.M. 1990, S. 393. Man beachte auch hier das Ineinander von akustischen und optischen Qualifizierungen.

21 Vgl. Franz Kafka: Tagebücher, S. 87f. (Oktober 1911).

22 Im berühmten Brief an den Vater erklärt er, dass er diesen zum "Maß aller Dinge« gemacht habe. Franz Kafka: Nachgelassene Schriften und Fragmente II, hg. von Jost Schillemeit, Frankfurt a.M. 1992, S. 143-217, hier S. 153 und 155f.

23 S.u. das Zitat aus Kafkas Tagebuchaufzeichnung vom Herbst 1911.

24 Vgl. Franz Kafka: Briefe 1913-März 1914, S. 134-135, hier S. 134. Kafkas Neigung, andere Personen nachzuahmen, hat auch in manchen seiner Prosastücke Spuren hinterlassen. Hier sei nur an den "Ausweg" erinnert, den Rotpeter im Bericht fï eine Akademie sucht, um nicht zugrunde zu gehen: "Es war so leicht, die Leute [die Besatzung auf dem Schiff, U.St.] nachzuahmen." Franz Kafka: Drucke zu Lebzeiten, hg. von Wolf Kittler u.a., Frankfurt a.M. 1994, S. 299-313, hier S. 308.

25 Im folgenden Zitat wird zwar auch das optische Moment hervorgehoben, indem von "Zuschauern« die Rede ist, die ihn (Kafka) mit Tucholsky hätten verwechseln können. Schon im nächsten Satz aber wird die Sichtbarkeit relativiert, indem das "fremde Wesen«, also in diesem Fall Tucholsky, »deutlich und unsichtbar « zunächst im eigenen (in Kafka) hätte existieren müssen. 
Safranski [!] [...] macht während des Zeichnens und Beobachtens Grimassen, die mit dem Gezeichneten [nämlich mit Tucholsky, U.St.] in Verbindung stehn. [Das] [e]rinnert mich daran, daß ich für meinen Teil eine starke Verwandlungsfähigkeit habe, die niemand bemerkt. Wie oft mußte ich Max [Brod] nachmachen. Gestern abend auf dem Nachhauseweg hätte ich mich als Zuschauer mit Tucholski [!] verwechseln können. Das fremde Wesen muß dann in mir so deutlich und unsichtbar sein, wie das Versteckte in einem Vexierbild, in dem man auch niemals etwas finden würde, wenn man nicht wüßte[,] daß es drin steckt. Bei diesen Verwandlungen möchte ich besonders gern an ein Sichtrüben der eigenen Augen glauben. ${ }^{26}$

Diese Eintragung hat mich schon einmal beschäftigt. ${ }^{27}$ Jetzt sollen die Erkenntnisse von damals aufgegriffen und fortgeführt werden. Angesichts der Verwandlung des zeichnenden Szafranski bemerkt Kafka, dass auch er sich in Tucholsky bis zur Verwechslung verwandelt habe. Er insistiert nicht auf seiner eigenen Identität - im Gegenteil, er gibt sie nolens volens preis, um sich selbst die des Anderen aneignen zu können. Der Tagebuchschreiber erinnert sich, dass er auch Brod schon häufig habe nachmachen müssen, und registriert obendrein, dass seine eigene Verwandlungsfähigkeit bislang unentdeckt geblieben sei. Mit dem Modell des Vexierbilds versucht er, sich den Übertragungsvorgang zu erklären. Er unterscheidet zwei Wesen, das eigene und das fremde. Letzteres sei »deutlich« ausgeprägt und doch zugleich "unsichtbar", d.h. sinnlich wahrnehmbar, aber unbemerkt, verborgen wie in einem Vexierbild. Man könne es nur entdecken, wenn man vorgängig von der Existenz des Fremden wisse und bereit sei, vom eigenen Wesen abzusehen. Die Existenz des einen wird an die Nichtexistenz des anderen gebunden, und das Gleiche gilt vice versa. ${ }^{28}$ Was sich als Ausschluss der jeweils anderen Instanz darstellt, lässt sich auch als exklusiver Wechsel zweier Befindlichkeiten fassen und in optischen Kategorien ausdrücken: Ein Bild muss verschwommen oder verworren geworden sein, damit aus seiner Undeutlichkeit eine deutliche Gestalt hervortreten kann. Nichts anderes meint der letzte zitierte Satz, der von einer Trübung des Augensinns spricht, die

26 Franz Kafka: Tagebücher, S. 46f.

27 Ulrich Stadler: «Mit Menschenverstand nicht mehr zu begreifen?« Über Missverständnisse und Unverständnisse der Prosa Franz Kafkas, in: Kannitverstan. Bausteine zu einer nachbabylonischen Herme(neu)tik, hg. von André Schnyder, München 2013, S. 387-396. Die Gestaltpsychologie hat diese wechselseitige Ausschließlichkeit beim Vexierbild als exklusives Verhältnis von Figur und (Hinter-)Grund beschrieben; vgl. E. Bruce Goldstein: Wahrnehmungspsychologie. Der Grundkurs, 9. Aufl., hg. von Karl R. Gegenfurtner, dt. von Katharina Neuser-von Oettingen und Guido Plata, Berlin/Heidelberg 2015, S. 109. Den Hinweis verdanke ich Wolfgang Marx, Zürich. 
bei solchen Verwandlungen vorausgesetzt werden müsse. Kafka formuliert ihn als Wunsch, denn er möchte das bisher Versteckte ausfindig machen.

Damit ist der sybillinische Bericht an Brod über das Bassermann-Erlebnis im Deutschen Theater ein wenig verständlicher geworden. Dem Umstieg von der einen Sichtweise auf die andere entspricht bei der Hamlet-Aufführung die Umpolung, die jeweils im Moment erfolgt, da der Theaterbesucher den Blick abwendet von der Bühne in die leere Loge. Der Kartenschreiber deutet das Iterative des Verwandlungsvorgangs an, ${ }^{29}$ indem er von mehreren Zeitabschnitten, nämlich von "ganzen Viertelstunden« und »von Zeit zu Zeit« spricht. Offensichtlich musste immer wieder ein Bruch inszeniert werden, damit das jeweils andere Bild zum Vorschein kommen konnte. Es entstand ein shifting zwischen zwei Identitäten und Zuständen, das sich mehrfach wiederholte.

Offen bleibt freilich immer noch, was »in Ordnung kommen« heißen soll. Befriedigend jedenfalls kann keiner der beiden exklusiven Positionen gewesen sein: Bassermännisch konnte Kafka nur werden, indem er sich selbst preisgab; als Theaterbesucher Franz Kafka konnte er sich nur erhalten, indem er seine eigene Bassermannhaftigkeit wieder abschüttelte. Beides zugleich ließ sich nicht realisieren.

Es ist darum kaum verwunderlich, dass Kafka sich selbst zwischen zwei Extrempunkten verortet hat. Er schwanke - so gesteht er zerknirscht seiner späteren Verlobten Felice Bauer - zwischen Nichtigkeit und einer Auffüllung seiner Existenz mit »etwas«. Daraus leitet er die vollständige Wertlosigkeit seines Urteilsvermögens ab:

Ich ruhe eben nicht in mir, ich bin nicht immer "etwas « und wenn ich einmal "etwas" war, bezahle ich es mit dem "Nichtsein« von Monaten. Darunter leidet natürlich [...] auch meine Menschenbeurteilung und meine Beurteilung der Welt überhaupt; ein großer Teil des für mich trostlosen Aussehns der Welt ist durch dieses schiefe Urteil veranlaßt [...]. Um es Dir an einem beliebigen Beispiel zu zeigen $[\ldots] .^{30}$

Und dieses »beliebige Beispiel« ist dann: Albert Bassermann, dessen Fotografien aus dem Film Der Andere er im Vorraum des Prager Kinos Bio Lucerna gesehen hat. Weil er offensichtlich wieder einmal unter 'Nichtsein litt, konnten die Bilder die Bassermann-Nähe in ihm hervorbringen. Sie sei

29 Das sich ja auch vor den Schaukästen des Kinos Bio Lucerna (s.u.) beobachten lässt.

30 Brief an Felice Bauer vom 4./5. März 1913, in: Franz Kafka: Briefe 1913-März 1914, S. 120-122, hier S. 120 f. 
aber dann wieder verschwunden, so dass sein Urteil über den Schauspieler insgesamt gelitten habe und "falsch" geworden sei. ${ }^{31}$ Der Brief geht in eine Todesphantasie über und schließt mit der Bekundung des Verlangens nach Zärtlichkeit in effigie gegenüber Felice Bauer im fernen Berlin - einer Zärtlichkeit, die Kafka später als eine von "Gespenstern" erschienen ist. ${ }^{32}$ Die Frage am Schluss des Briefes kehrt das shifting in der Beurteilung der eignen Person wie der Geliebten noch einmal eindrucksvoll hervor:

Wenn er sich einmal aufheben wird [d.h. wenn Bassermann einmal sterben wird, U.St.] [,] wird er sich eben ganz aufheben und nicht mehr dasein, ${ }^{33}$ aber nicht wie ich es tue und jedem es andichten möchte, mich immerfort umfliegen wie ein Vogel, der durch irgendeinen Fluch von seinem Neste abgehalten, dieses gänzlich leere Nest immerfort umfliegt und niemals aus den Augen läßt.

Gute Nacht, Liebste. Darf ich Dich küssen, darf ich den wirklichen Körper umarmen?

Franz $^{34}$

2.

Es bleibt noch zu klären, welche Bedeutung der Differenzierung von Sehen und Hören in der kurzen Mitteilung an Brod zukommt. Es sei daran erinnert, dass der Postkartenschreiber Kafka befand, den Hamlet Bassermanns gehört zu haben, sei die bessere Formulierung, als ihn gesehen zu haben. Damit war - wohl gemerkt - nicht gesagt, dass das Hören der richtige und das Sehen der falsche Ausdruck wäre, sondern nur dass die akustische der optischen Terminologie vorzuziehen sei. Der Komparativ »besser« nimmt

31 Ebd., S. 122.

32 Gegenüber einer anderen Geliebten, nämlich Milena Jesenská, bezeichnete Kafka Briefwechsel generell als »Verkehr mit Gespenstern" und behauptete: "Geschriebene Küsse kommen nicht an ihren Ort, sondern werden von den Gespenstern auf dem Wege ausgetrunken." Franz Kafka: Briefe an Milena. Erweiterte und neu geordnete Ausgabe, hg. von Jürgen Born und Michael Müller, Frankfurt a.M. 1983, S. 302. Zu geschriebenen Küssen und dergleichen vgl. meinen demnächst erscheinenden Aufsatz: Ulrich Stadler: Schreiben, umschreiben und vorlesen. Über Kafkas Umgang mit der Erzählung "Das Urteil«, in: Lesen, schreiben, edieren. Über den Umgang mit Literatur, hg. von Peter Kofler und Ulrich Stadler, Frankfurt a.M./Basel 2016, S. 43-63.

33 Die Selbstsicherheit, die Kafka hier Bassermann zuspricht, geht wohl zurück auf den Bericht, den der Schauspieler über sich und seine Rolle im Film Der Andere verfasst hat. Vgl. Albert Bassermann: Wie ich mich im Film sehe, S. 33f.

Franz Kafka: Briefe 1913-März 1914, S. 122. 
eine relative Bewertung vor, impliziert aber auch, dass keiner der beiden Ausdrücke die Sache vollkommen erfasst. Die Höherschätzung des Auditiven widerspricht der in Europa seit Beginn der Frühen Neuzeit fast uneingeschränkt geltenden »okularen Tyrannis« ${ }^{35}$ Der Vorherrschaft des Optischen ist allerdings auch schon um 1800 widersprochen worden. ${ }^{36}$ Walter J. Ong hat im 20. Jahrhundert die Vorzüge des Gehörs darin gesehen, dass es wie kein anderes Sinnesorgan geeignet sei, »das Innere eines Objekts zu erfassen ${ }^{37}{ }^{37}$ Und im Unterschied zum Sehen, das zergliedere und isoliere, sei das Hören "ein vereinender Sinn « ${ }^{38}$ Wenn sich diese Einschätzungen auch, wie noch deutlicher werden wird, durchaus auf den Fall des Kartenschreibers Kafka beziehen lassen, so ist doch Vorsicht geboten. Ongs Darstellung ist problematisch - nicht so sehr, weil er dazu neigt, die menschlichen Wahrnehmungsvermögen zu anthropologischen Konstanten zu verfestigen, sondern auch und vor allem deshalb, weil er das Verhältnis von gesprochener Sprache und Schrift, von Oralität und Literalität allzu sehr als einen fundamentalen Gegensatz begreift. ${ }^{39}$

35 Ulrich Sonnemann: [Interview mit Florian Rötzer], in: Denken, das an der Zeit ist, hg. von Florian Rötzer, Frankfurt a.M. 1987, S. 262-277, hier S. 277. Sonnemann stellt der Tradition der Okulartyrannis »eine ganz andere« gegenüber, nämlich eine, »die dem Judentum entspringt und viel mehr mit dem Ohr zu tun hat« (ebd.). Zu den ganz auf das Wahrnehmungsorgan Auge Fixierten gehörte zweifellos auch Lavater, der sich selbst in "Ansehung seiner Stimme« einer "gänzlichen Tonkunstunwissenheit« bezichtigte. Johann Caspar Lavater: Physiognomische Fragmente zur Beförderung der Menschenkenntniß und Menschenliebe, 4. Versuch, Leipzig/Winterthur 1778, S. 418. An den Ohren interessierte ihn nur die Form, nicht aber ihre Erkenntnisfunktion. In den vier Bänden seiner Physiognomischen Fragmente gibt es lediglich eine einzige kleine Fußnote (ebd.), die auf die spezifische Leistung des Gehörs Bezug nimmt.

36 Über die keineswegs einheitliche Situation unterrichtet das Buch von Peter Utz: Das Auge und das Ohr im Text. Literarische Sinneswahrnehmung in der Goethezeit, München 1990. Ergänzend sei hier auf die einzigartige Stellungnahme Ritters hingewiesen, die sich scharf von der Hochschätzung des Auges bei Novalis unterscheidet. Vgl. Johann Wilhelm Ritter: Fragmente aus dem Nachlasse eines jungen Physikers [1810], hg. von Heinrich Schipperges, Heidelberg 1969, Bd. 1, S. 223f. (Nr. 358).

37 Walter J. Ong: Oralität und Literalität. Die Technologisierung des Wortes, aus dem Amerikanischen von Wolfgang Schömel, Opladen 1987, S. 74. Den Hinweis auf das Buch von Ong verdanke ich Peter Utz, Lausanne.

38 Ebd., S. 75.

39 Zur Kritik dieses sphonographischen Dogmas` vgl. vor allem Sybille Krämer: Die Schrift als Hybrid aus Sprache und Bild. Thesen über die Schriftbildlichkeit unter Berücksichtigung von Diagrammatik und Kartographie, in: Bilder. Ein (neues) Leitmedium?, hg. von Torsten Hoffmann und Gabriele Rippl, Göttingen 2006, S. 79-92, hier S. $79 f$. 
Der 1883 in Prag geborene Franz Kafka hat sich sehr intensiv mit dem Verhältnis von Gesicht und Gehör beschäftigt, und zwar aus zwei verschiedenen, aber letztlich einander zuarbeitenden Motiven und Veranlassungen heraus.

Zum einen hat er, der prinzipiell, trotz mancher gegenteiligen Äußerung, seine Werke veröffentlichen wollte, sofern sie in seinen Augen einigermaßen gelungen waren ${ }^{40}$ auf doppelte Weise die Publizität gesucht. Er hat zumindest einige Texte, wenn auch nach großen Widerständen, ${ }^{41}$ drucken lassen - im Zusammenhang mit der Betrachtung gesteht er Rowohlt etwa seine "Gier, unter Ihren Büchern auch ein Buch zu haben « ${ }^{42}$-, die Verkaufszahlen seines Erstlings waren allerdings trotz durchwegs guter Kritiken gering und das Buch ein »totaler finanzieller Mißerfolg « ${ }^{43}$ Obendrein aber hat er seine Texte sehr gerne vor ausgewählten Zuhörern und Zuhörerinnen laut vorgelesen. Er suchte also Publizität, indem er seine Texte sowohl sichtbar als auch hörbar machte. Seine Vortragskunst ist von Lothar Müller untersucht und in die Tradition der Dichterlesungen und Deklamationsveranstaltungen seit Goethe und Tieck gestellt worden. ${ }^{44}$ Dabei kommt Müller zu dem Schluss, dass bei Kafka eine besonders enge "Koppelung von Stimme und Buch" vorliege..$^{45}$ Die Oralität ist fragiler und störanfälliger als die Literalität; sie ist auf einen individuellen, lebendigen Übermittlungsvorgang angewiesen, den der Sprecher zu seinem Publikum herstellen muss. Kafka hat sich in den Berichten über seine eigenen Lesungen immer wieder gefragt, ob es ihm gelungen sei, die Distanz zwischen sich und dem Publikum zu überwinden. Er hat über das gesprochene Wort eine Art Vereinigung mit seinen Hörern und Hörerinnen angestrebt, die durchaus erotisch-sexuell konnotiert gewesen ist. ${ }^{46}$ Freilich benötigte er hierfür immer auch das geschriebene Wort:

40 Das ist jedenfalls das Fazit der sorgfältigen Untersuchung von Joachim Unseld: Franz Kafka. Ein Schriftstellerleben. Die Geschichte seiner Veröffentlichungen, München 1982, S. 144, 152, 178, 245 u.ö.

41 Man vergleiche dazu etwa die Druckgeschichte der Betrachtung: Franz Kafka: Drucke zu Lebzeiten, Apparatband, S. 37 und 40.

42 Ebd., S. 40.

43 Hartmut Binder: Geschichte der Kafka-Rezeption. Frühphasen der Kritik, in: KafkaHandbuch, hg. von dems., Stuttgart 1979, S. 583-624, hier S. 585.

44 Vgl. Lothar Müller: Die zweite Stimme. Vortragskunst von Goethe bis Kafka, Berlin 2007.

45 Vgl. ebd., S. 71, 91 und 99-101.

46 Schon Herder hatte in einem Horen-Beitrag über Homer als Sänger den Rhapsoden als einen Sänger oder einen »Sprechenden« bezeichnet, der es darauf anlege, mit seinen Zuhörern "gleichsam ganz Eins zu werden. "Johann Gottfried Herder: Sämmtliche Werke, hg. von Bernhard Suphan, Bd. 18, Berlin 1883, S. 424-427, hier S. 426. Zu den Vereinigungs- 
Er trug nur vor, wenn er ein Manuskript oder ein Typoskript zur Verfügung hatte. Die Rede war für ihn nicht identisch mit der Schrift, wohl aber benötigte sie diese als Grundlage.

Zum andern hat Franz Kafka neben seinem Jura-Studium an der FerdinandKarls-Universität auch Lehrveranstaltungen anderer Fächer besucht und überhaupt rege am kulturellen Leben der Stadt teilgenommen. Er war mit Franz von Brentanos Lehre von der "analysierende[n] Beschreibung [der] Gegenstände, welche wir in unserer Wahrnehmung erfassen ${ }^{47}{ }^{47}$ vertraut; d.h., die sdeskriptive Psychologie war auch ihm ein Begriff, wenn er auch ihr gegenüber wohl kritisch eingestellt war. ${ }^{48}$ Was Brentano über die Sinne und ihr Verhältnis zueinander, über den Zusammenhang von Phantasie- und Wahrnehmungsvorstellungen, über optische Täuschungen ${ }^{49}$ und Mitempfindungen geschrieben hatte, wurde von seinen Schülern in Prag, Wien, Graz und Berlin aufgegriffen und differenziert. Kafka kannte die einschlägigen Diskussionen von den Zusammenkünften der Prager Brentanisten im Cafe Louvre $^{50}$ und auch von den Diskussionsabenden im Hause von Berta und Max Fanta. Der eigenwilligste Brentano-Schüler unter Fantas Gästen war Christian von Ehrenfels, dem Kafka sich besonders verbunden fühlte - nicht

bzw. Verschmelzungswünschen Kafkas bei seinen Lesungen vgl. meinen Aufsatz: Ulrich Stadler: Schreiben, umschreiben und vorlesen, S. $62 \mathrm{f}$.

47 Franz Brentano: Deskriptive Psychologie, aus dem Nachlaß hg. und eingeleitet von Roderick M. Chisholm und Wilhelm Baumgartner, Hamburg 1982, S. 130.

48 Vgl. Franz Kafka: Nachgelassene Schriften und Fragmente II, Apparatband, S. 199. Die von Kafka wiederholt geäußerte und in der Kafka-Literatur häufig zitierte Maxime "Zum letzten Mal Psychologie!« (ebd., Textband, S. 81, 134) wird zumeist allzu rasch mit der Lehre Freuds in Verbindung gebracht. Was Kafka allerdings jeweils unter >Psychologier verstand, ist nicht so einfach zu entschlüsseln. Es sei daran erinnert, dass spsychologisch in der Zeit um und nach 1900 beinahe identisch gesetzt werden konnte mit ästhetisch. Theodor Lipps etwa behauptet kategorisch, dass eine ästhetische Untersuchung "immer psychologisch" sei. Theodor Lipps: Komik und Humor. Eine psychologische-ästhetische Untersuchung, Hamburg/Leipzig 1898, S. 7. Und der unten erwähnte Felix Weltsch hält in seinem Referat über Henri Bergson in der Bohemia vom 13. März 1913, das zugleich eine Propagandaschrift für das von ihm und Max Brod geschriebene Buch Anschauung und Begriff darstellt, eine "aufmerksame und peinliche Erforschung der zugrundeliegenden psychischen Phänomene « für unabdingbar. Franz Kafka: Briefe 1913-März 1914, S. 434-438, hier S. 438.

49 Vgl. die Abhandlungen Brentanos über Optische Paradoxien, in: Franz Brentano: Untersuchungen zur Sinnespsychologie, 2. durchges. Aufl., hg. von Roderick M. Chisholm und Reinhard Fabian, Hamburg 1979. Zu den Mach'schen Bändern vgl. E. Bruce Goldstein: Wahrnehmungspsychologie, S. 55 und 246.

50 Dort versammelte sich alle vierzehn Tage ein Philosophenzirkel, in dem »die Lehre Franz Brentanos beinahe unumschränkt herrschte«, wie Brod berichtet. Max Brod: Streitbares Leben 1884-1968, S. 164. 
zuletzt weil er neben seiner Tätigkeit als ordentlicher Professor für Philosophie an der Prager Universität auch ausgesprochen künstlerische Interessen verfolgte. Ehrenfels hatte mit seiner Schrift Über `Gestaltqualitäten`von 1890, die ihm den Ruf an die Prager Universität eintrug und die ihn damit »in das Zentrum der Brentano-Schule« versetzte, ${ }^{51}$ eine wahrnehmungspsychologische Begründung des aristotelischen Diktums geliefert, wonach das Ganze mehr, ja etwas anderes sei als die Summe seiner einzelnen Teile. ${ }^{52}$ Bei seiner Argumentation hatte er sich - und das ist nun bezeichnend - vor allem auf Belege aus der Musik gestützt. Er hatte gezeigt, dass eine Melodie etwas grundsätzlich anderes sei als die Töne, aus denen sie sich zusammensetzt. ${ }^{53}$ Die Melodie fasste er als `Gestaltqualität‘, während die einzelnen Töne, aus denen sie besteht, als voneinander trennbare Elemente, bloß die Grundlage der jeweiligen Gestaltqualität darstellten. Diese hafte als simultanes Gebilde besser im Gedächtnis als die einfachen Elemente. ${ }^{54}$ An ihrer Entstehung seien innere Vermögen maßgeblich beteiligt, und zwar in einer Weise, die sich dem Bewusstsein des aufmerkenden Gemüts entziehe. Nur so viel steht für Ehrenfels fest: Bei der Zusammenfassung einzelner zeitlich gesonderter Elemente zu einem Gesamtbild sei das Gehör dem Gesicht »weit überlegen «. ${ }^{5}$ Während der Blick sich an die Oberfläche hält und mit ihr vorliebnehmen muss, durchdringt sie der Ton und findet den Weg von der eigenen Innerlichkeit zu der des Gegenübers scheinbar mühelos. ${ }^{56}$ Wenn er

51 So Ehrenfels selbst in der Erinnerung, mit der er seine "Weiterführenden Bemerkungen« zu seiner berühmtesten Schrift von 1922 einleitete. Christian von Ehrenfels: Über `Gestaltqualitäten<, in: Psychologie, Ethik, Erkenntnistheorie. Philosophische Schriften, Bd. 3, hg. von Reinhard Fabian, München u.a. 1988, S. 128-167, hier S. 155.

52 Vgl. ebd., S. 134, und Aristoteles: Metaphysik, hg. von Friedrich Bassenge, Buch Z, Kap. 17, Berlin 1960, S. 189 [1041b]. Ehrenfels hat selbst seinen Begriff der `Gestaltqualitäten mit dem der `Ganzheiten in Verbindung gebracht. Vgl. Christian von Ehrenfels: Über `Gestaltqualitäten<, S. 151, 154, 157 und 160.

53 Ehrenfels glaubte "unwiderleglich" gezeigt zu haben, "daß die Melodie oder Tongestalt etwas anderes ist als die Summe der einzelnen Teile, auf welchen sie aufbaut« (ebd., S. 134).

54 Für Bergson wäre das kaum verwunderlich, denn dasjenige, was Ehrenfels die "Gestaltqualität" nennt, ist bei ihm auch und vor allem ein Produkt der erinnernden Arbeit des Gedächtnisses. Vgl. Henri Bergson: Materie und Gedächtnis. Eine Abhandlung über die Beziehung zwischen Körper und Geist, neu übersetzt von Julius Frankenberger, 2. Aufl., Jena 1919, S. 18, 55 und 121.

56 Ong zufolge zeichnet sich das Hören gegenüber dem Sehen dadurch aus, dass es Interiorität registrieren kann, ohne sie zu beschädigen: »Klänge registrieren stets die innere Beschaffenheit der Dinge, die sie hervorbringen. [...] Die menschliche Stimme kommt aus dem Inneren des menschlichen Körpers, der die Resonanz der Stimme bildet. Das Sehen isoliert, das Hören bezieht ein. Während das Sehen den Beobachter außerhalb des 
leichter als der Blick ins Innere dringen kann, dann kann er auch leichter bei der Vorstellung eines Ganzen mitwirken. Dieser Auffassung war jedenfalls Henri Bergson, dessen Matière et mémoire aus dem Jahre 1896 Kafka und seinen Freunden vertraut gewesen ist. ${ }^{57}$

Wenn also auch die einzelnen sichtbaren Wahrnehmungsvorstellungen von anderer Beschaffenheit sein mögen als die hörbaren, so verflüchtigt sich doch für Ehrenfels diese Differenz bei den jeweiligen Gestaltqualitäten. Der Melodie entspreche das »Bild«:58 Auch bei einem solchen wirkten die Erinnerung und die Phantasietätigkeit des Betrachters mit, und auch hier entstehe etwas »Neues«. ${ }^{59}$ Für Ehrenfels lag daher der Schluss nahe, dass seine Lehre von den Gestaltqualitäten geeignet sei, "die Kluft zwischen den verschiedenen Sinnesgebieten « - also auch die zwischen dem Gehör- und dem Gesichtssinn! - »zu überbrücken und die anscheinend disparatesten Erscheinungen unter ein einheitliches System zusammenzufassen«. .0

Die Schrift Über >Gestaltqualitäten` ist wie Bergsons Materie und Gedächtnis im Freundeskreis Kafkas intensiv rezipiert worden. Ein Beleg für diese Rezeption stellt das Buch Anschaunng und Begriff von Felix Weltsch und Max Brod dar. Kafka war ganz sicher mit der Thematik dieses Buches seiner engsten Freunde vertraut, längst bevor es im Frühjahr 1913 erschien. Er las es wiederholt, ${ }^{61}$ er kannte auch die zusammenfassende Darstellung von Felix Weltsch in der Bohemia vom 13. März $1913^{62}$ und besuchte obendrein mehrere Sitzungen eines Seminars von Christian Ehrenfels, in denen es

Betrachteten hält, dringt ein Klang in den Hörer ein. [...] Im Gegensatz zum Sehen, dem zergliedernden Sinn, ist somit das Hören ein vereinender Sinn.« Walter J. Ong: Oralität und Literalität. Die Technologisierung des Wortes, S. 75.

57 Brod und Weltsch stützen sich in ihrer, auch Kafka bekannten Untersuchung über die Entstehung von Begriffen mit Vorliebe auf Bergsons Abhandlung. S.o. sowie Max Brod/ Felix Weltsch: Anschauung und Begriff. Grundzüge eines Systems der Begriffsbildung, Leipzig 1913, S. 154 und 161.

58 ’Bild im Sinne von Ehrenfels, nicht im Sinne von Bergson: »Was nämlich hier [bei der Betrachtung eines Bildes, U.St.] durch die Empfindung gegeben wird, ist keineswegs jene Vorstellung, welche der Maler durch das Bild zu vermitteln suchte, sondern nur ein ärmliches Skelett, um welches sich jene durch Phantasietätigkeit erst heranbilden muß.« Christian von Ehrenfels: Über `Gestaltqualitäten`, S. 151.

59 Ebd., S. 149.

60 Ebd., S. 153.

61 Die Argumentation schien ihm sehr abstrakt, jedenfalls bezeichnete er sie in einem Brief an Felice Bauer vom 27./28. Februar 1913 als »recht streng philosophisch«. Franz Kafka: Briefe 1913-März 1914, S. 112-113, hier S. 112. Vgl. auch den schon mehrfach zitierten Brief vom 5./6. März 1913 an Felice, in: Franz Kafka: Briefe 1913-März 1914, S. 122f.

62 Vgl. den Abdruck mit Kafkas handschriftlichen Korrekturen im Kommentarteil, in: Franz Kafka: Briefe 1913-März 1914, S. 434-438. 
diskutiert wurde. ${ }^{63}$ Wenn er im Tagebuch für sich phasenweise ein "Sichtrüben der eigenen Augen" erhofft, dann stimmt er mit Weltsch und Brod überein, die der Verschwommenheit in ihrem Buch eine zentrale Rolle als konstruktives Moment bei der Begriffsbildung zusprechen. Und wenn er sich im Postkartengruß korrigiert und lieber von 'gehört haben` als von 'gesehen haben spricht, dann gibt er Brod zu verstehen, dass ihm die Begegnung mit Bassermann nicht nur eine reines, d.h. eine vereinzelte, isolierte Wahrnehmung geliefert hat. Er versichert dem künftigen Co-Autor von Anschaunng und Begriffauch, dass der Hamlet-Darsteller für ihn etwas geworden ist, was Brod und Weltsch in ihrem Buch mit einem Begriff Bergsons ${ }^{64}$ als "allgemeine[s] Erinnerungsbild « ${ }^{65}$ bezeichnen sollten.

Kafka hat, soweit ich sehe, in seinen eigenen Arbeiten selten die Position und niemals längere Textpassagen eines anderen Autors vorbehaltlos und unverändert übernommen. Auch im vorliegenden Fall hat er dies nicht getan. Immerhin ist im Buch seiner Freunde bereits vom »Vexierbild« die Rede. ${ }^{66}$ Während Brod und Weltsch jedoch damit bloß betonen wollen, dass Deutbarkeit und Begriffsbildung auf Verschwommenheit angewiesen sind, ${ }^{67}$ interpretiert Kafka das Vexierbild auf seine Weise. Die im Brief an Felice Bauer etwas gar zu defätistische Klage über die absolute Urteilsunfähigkeit und Nichtigkeit der eigenen Existenz wird in der Tagebuchnotiz modifiziert. Die Aneignung fremder Gesichter beruht zwar auf der Bereitschaft, die eigene Nichtigkeit anzuerkennen; letztere ist aber keineswegs so »leer", wie Kafka in seiner buchstäblich vernichtenden Selbstkritik gegenüber der Freundin behauptet. Sie muss vielmehr all jene fremden Gesichter schon latent in sich enthalten, damit diese aus ihrer Virtualität heraustreten können. Insofern stellt das Vexierbild für ihn ein Modell seiner dichterischen Weltaneignung überhaupt dar. Denn nur in der Trübung, der Verschwommenheit des Wahrgenommenen, und in der Bereitschaft, das eigene Ich zurücktreten zu lassen, können die fremden Bilder als eigene Texte zum Vorschein kommen.

Harry Kahn hat zwar in seiner Schaubühnen-Rezension eine einigermaßen verlässliche Beschreibung des Bassermann'schen Hamlet geliefert, aber sein

63 Dem Urteil Brods zufolge soll sich Kafka dort »begeistert « gezeigt haben. Vgl. Franz Kafka: Briefe 1913-März 1914, S. 524, und Max Brod: Streitbares Leben 1884-1968, S. 164.

64 Vgl. Henri Bergson: Materie und Gedächtnis, S. 119f., 121f., 125 und 127f. Im französischen Original ist von »le souvenir-image« die Rede. Henri Bergson: Matière et mémoire. Essai sur la relation du corps à l'esprit, 6. Aufl., Paris 1910, S. 143 u.ö.

66 Ebd., S. 63.

67 Vgl. ebd. 
»Gestalt«-Begriff verdeckte den eignen subjektiven Anteil bei der Genese dessen, was er »die ganze Art Bassermanns« genannt hat. Kafka hingegen knüpfte offensichtlich an die Einsichten von Bergson und Ehrenfels an. Von jenem übernahm er den Grundsatz, dass bei jeder Wahrnehmung automatisch auch die Mitarbeit des Gedächtnisses vorausgesetzt werden müsse. Ehrenfels aber verdankte er die Einsicht, dass die "Gestaltqualität" sich maßgeblich der Phantasietätigkeit verdanke, deren Beteiligung allerdings auf glückhafte Momente angewiesen ist. Einen solchen glückhaften und mitteilenswerten Moment erlebte er in der Begegnung mit Bassermann. Sie lieferte ihm nicht nur eine einzelne Empfindung, »ein ärmliches Skelett", sondern ein Ganzes, in dem Shakespeares Stück und Reinhardts Inszenierung gleichermaßen aufgehoben waren. Kafka vermied den Terminus 'Gestaltqualität‘ und zog das anschaulichere Wort 'Gestalt ${ }^{68}$ oder den besonders vieldeutigen Begriff »Gesicht « ${ }^{69}$ vor. Unzweifelhaft aber macht sich in den zwei Sätzen auf der Briefkarte an Brod die Gestaltqualität des Hamlet-Darstellers Bassermann geltend. Sie entzog und entzieht sich der unmittelbaren Sichtbarkeit und Hörbarkeit, wenn sie auch auf dem beruhte, was das Gesicht und mehr noch das Gehör dem Theaterbesucher zugetragen hatten. Ihre geradezu schmerzhafte Deutlichkeit aber war und ist vor allem der Selbstaufgabe des Theaterbesuchers und Briefschreibers Franz Kafka geschuldet. Erst sie macht es möglich, dass wir Leser uns in Kafka wiederkennen, so wie sich dieser in Bassermann wiedererkannt hat.

68 In dem Prosastück Der plötzliche Spaziergang etwa ist am Schluss von der "wahren Gestalt« die Rede. Franz Kafka: Drucke zu Lebzeiten, S. 17-18, hier S. 18. Dass bei solcher Benennung auch Reminiszenzen an die Goethezeit bei Kafka eine Rolle gespielt haben könnten, soll damit keineswegs bestritten werden. Vgl. dazu Karl Pestalozzi: Spazieren und Schreiben - Franz Kafka "Der plötzliche Spaziergang« und Robert Walser »Der Spaziergang«, in: Franz Kafka und Robert Walser im Dialog, hg. von Vesna Kondrič Horvat, Berlin 2010, S. 23-39, hier S. $28 f$.

69 ,Gesicht bezeichnet erstens die Vorderseite eines Kopfes, zweitens analog zu Gehör ein Wahrnehmungsorgan, drittens ein wahrgenommenes oder wahrnehmbares Objekt als Gegenstand physiognomischer und pathognomischer Neugierde, viertens das sinnlich wahrnehmbare Identitätsmerkmal, d.h. so etwas wie die Etikette einer Person und fïnftens eine mystische Schau, eine Vision. Alle diese Bedeutungen - mit Ausnahme vielleicht der letzten - finden sich bei Kafka, und häufig lassen sie sich nicht scharf voneinander trennen. Der Ausdruck war im Übrigen nicht nur bei Kafka beliebt, auch andere Autoren aus den ersten drei Jahrzehnten des 20. Jahrhunderts verwendeten den Begriff mit Vorliebe, wobei sie ihn nicht nur auf einzelne Menschen, sondern überindividuell auf Sachverhalte und Zustände bezogen. Vgl. den Beitrag von Peter Utz im vorliegenden Band. 
